\title{
GENERATORS AND STRONG GENERATORS IN ERGODIC THEORY
}

\author{
BY WILLIAM PARRY
}

Communicated by P. R. Halmos, November 15, 1965

The purpose of this note is to answer in the affirmative the question $^{1}$ raised by Rohlin [1], [2] as to whether every ergodic automorphism of a Lebesgue space with continuous measure possesses a countable generator. Rohlin has dealt with the case of automorphisms with finite entropy [2], and for this case he proved the existence of a countable generator with finite entropy. Automorphisms with infinite entropy such as the Bernoulli shift on a doubly infinite product of unit intervals were not amenable to Rohlin's method. It is well known that a generator of an automorphism with zero entropy is also a strong generator. Our result actually produces strong generators for arbitrary ergodic nonsingular one-one transformations, and thus, for the case of automorphisms with positive entropy these strong generators have infinite entropy. ${ }^{2}$

Rohlin imposed the weaker condition of aperiodicity rather than ergodicity and our result can be proved under this condition also, but in order to avoid undue emphasis on technicalities, we will assume that $T$ is an ergodic nonsingular one-one transformation of a Lebesgue space $(X, \Theta, m)$ with continuous measure such as the unit interval. Our result implies that every ergodic automorphism can be viewed as the shift associated with a stationary stochastic process (strong sense) with a countable number of states.

For the basic properties of measurable partitions and subalgebras cf. $[1],[3]$.

A countable measurable partition $\zeta$ of $X$ is called a generator [strong generator] if

$$
\bigvee_{n=-\infty}^{\infty} T^{n} \zeta=\Theta\left[\bigvee_{n=0}^{\infty} T^{-n} \zeta=\Theta\right]
$$

${ }^{1}$ Since submitting this note V. A. Rohlin has drawn my attention to his paper Generators in ergodic theory. II, Vestnik Leningrad. Univ. Mat. Meh. Astronom. 13 (1965), 68-72 (Russian), which contains the main result presented here. However, the two proofs are entirely different.

${ }^{2}$ I am grateful to David A. Freedman for communicating to me a proof of the existence of a strong generator for the two shift. It was this communication which provoked the work presented here. 
or equivalently if there exists a set $M$ of measure zero such that $x, y \in T^{-n} C_{n}, \quad x, y \in X-M, \quad C_{n} \in \zeta(n=0, \pm 1, \pm 2, \cdots)$

$$
[n=0,1,2, \cdots]
$$

implies $x=y$.

Leмma. There exists a countably infinite measurable partition $\alpha=(A(0), A(1), \cdots)(m(A(i))>0, i=0,1, \cdots)$, a sequence $\left\{a_{n}\right\}$ of positive integers, and set $M$ of measure zero such that if

$$
f(x)=\sum_{n=0}^{\infty} a_{n} \chi_{A(n)}(x)
$$

then $\lim \sup _{n \rightarrow \infty} f\left(T^{n} x\right)-n=+\infty$ for all $x \in X-M$.

Proof. Let $\alpha=(A(0), A(1), \cdots)$ be any countably infinite measurable partition. Using the ergodicity and nonsingularity of $T$ one can show that

$$
m\left(\bigvee_{k=0}^{\infty} T^{-k} A(n)\right)=1, \quad n=0,1, \cdots
$$

Choose an increasing sequence $\{N(n)\}$ of positive integers such that $m\left(\cup_{k=0}^{N(n)} T^{-k} A(n)\right) \rightarrow 1$. There is a set $M$ of measure zero such that if $x \in X-M, x$ belongs to infinitely many of the sets $\bigcup_{k=0}^{N(n)} T^{-k} A(n)$, i.e. $x \in \bigcup_{k=0}^{N(j)} T^{-k} A(j), j \in J$ where $J$ is an infinite set of nonnegative integers.

In other words for every $j \in J$ there is an integer $k(j), 0 \leqq k(j)$ $\leqq N(j)$ such that

$$
T^{k(j)} \in A(j)
$$

Define $a_{n}=2 N(n)$ and $f(x)=\sum_{n=1}^{\infty} a_{n} \chi_{A(n)}(x)$ then $f\left(T^{k(j)} x\right)-k(j)$ $=a_{j}-k(j)=2 N(j)-k(j) \geqq N(j)$. Consequently

$$
\limsup _{n \rightarrow \infty} f\left(T^{n} x\right)-n=+\infty \quad \text { if } x \in X-M \text {. }
$$

TheOREM. $T$ possesses a strong generator.

Proof. Let $\delta_{(0)} \leqq \delta_{(1)} \leqq \delta_{(2)} \leqq \cdots$ be countably infinite measurable partitions converging to the unit partition $\epsilon$ consisting of single point sets,

$$
\delta_{(n)} \rightarrow \epsilon .
$$

Let $\epsilon_{(0)}=\delta_{(0)}, \epsilon_{(n)}=\delta_{(n)} \bigvee T \delta_{(n)} \bigvee \cdots \bigvee T^{n} \delta_{(n)}$ then 


$$
\epsilon_{(0)} \leqq \epsilon_{(1)} \leqq \epsilon_{(2)} \leqq \cdots \cdot
$$

are also countably infinite measurable partitions converging to $\epsilon$. Moreover

$$
T^{-k} \epsilon_{(n)} \geqq \epsilon_{(n-k)} \quad(n \geqq k) .
$$

Let $\epsilon_{(n)}=\left(A_{0}^{n}, A_{1}^{n} \cdots\right)$ and let $f=\sum a_{n} \chi_{A_{n}^{0}}$ be the function defined in the lemma when $\alpha=\epsilon_{(0)}$.

Let $A_{\mathrm{k}}^{0} \cap \epsilon\left(a_{k}\right)$ be the countable partition of $A_{\mathrm{k}}^{0}$ consisting of sets $A_{\boldsymbol{k}}^{0} \cap B, B \in \epsilon\left(a_{k}\right)$, and let $\zeta=\bigcup_{k=0}^{\infty} A_{k}^{0} \cap \epsilon\left(a_{k}\right)$ so that $\zeta$ is a countable measurable partition of $X$. We will show that $\zeta$ is a strong generator of $T$ by showing that if $x, y \in X-M$ (where $M$ is the set of measure zero in the lemma) and if $x, y \in T^{-n} C_{n}, C_{n} \in \zeta(n=0,1, \cdots)$, then $x=y$.

Let $\theta(n)=f\left(T^{k(n)}(x)\right)-k(n) \uparrow+\infty$.

$$
f\left(T^{k(n)} x\right)-k(n)=a_{j}-k(n) \text { if } T^{k(n)} x \in A_{j}^{0} .
$$

But $T^{k(n)} x \in C_{k(n)}$ and therefore $C_{k(n)}$ has the form $C_{k(n)}=A_{j}^{0} \cap B$ where $B \in \epsilon(a(j))$

i.e. $x, y \in T^{-k(n)} C_{k(n)} \subset T^{-k(n)} B \subset B^{\prime}$ for some $B^{\prime} \in \epsilon(a(j)-k(n))=\epsilon(\theta(n))$.

We have shown that $x, y$ belong to the same elements of $\epsilon(n)$ for infinitely many $n$ and consequently $x, y$ belong to the same elements of $\epsilon(n)$ for every $n$. Since $\epsilon(n) \uparrow \epsilon, x=y$.

REMARK. A strong generator for $T$ is not necessarily a strong generator for $T^{-1}$, (examples exist) but by refining a strong generator for $T$ with a strong generator for $T^{-1}$ one obtains a partition which is simultaneously a strong generator for $T$ and $T^{-1}$.

\section{REFERENCES}

1. V. A. Rohlin, New progress in the theory of transformation with invariant measure, Uspehi Mat. Nauk 15 (1960), 3-26=Russian Math. Surveys 15 (1960), 1-22.

2. - Generators in ergodic theory, Vestnik Leningrad. Univ. Ser. Mat. Meh. Astronom. (1963), 26-32. (Russian)

3. - On the fundamental ideas of measure theory, Mat. Sb. 25 (1949), 107$150=$ Amer. Math. Soc. Transl. (1) 10 (1962), 1-54.

UNIVERSITY OF SUSSEX 